\title{
INFLUENCE DE L'HYDRODYNAMIQUE DES BASSINS SUR LA SURVIE ET LA CROISSANCE DES LARVES DE TURBOT (PSETTA MAXIMA).
}

\author{
J.L. GAIGNON, B. PETTON et L. QUÉMÉNER
}

IFREMER, Laboratoire de Physiologie des Poissons, BP 70, 29280 Plouzané, France.

\section{RÉSUMÉ}

Une forte variabilité des performances larvaires est observée chez le turbot. De nombreuses causes ont été étudiées, mais peu de travaux ont été publiés sur l'influence des conditions environnementales. Dans cet article, les effets des conditions hydrodynamiques des milieux d'élevage sur la survie et la croissance de larves nourries jusqu'aux jours $7-10$ et de larves non alimentées ont été étudiés. Plusieurs expérimentations ont porté sur l'importance des débits d'eau (600 et $1200 \mathrm{ml} \cdot \mathrm{mn}^{-1}$, constant et progressif), des débits d'air (10 et $30 \mathrm{ml}^{\mathrm{m}} \mathrm{mn}^{-1}$ ) et du sens de circulation de l'eau (descendant et ascendant, avec et sans changement de sens en cours d'élevage). De plus, les interactions possibles du sens de circulation de l'eau avec les conditions d'alimentation des larves ont été étudiées (i) en différant la première distribution alimentaire (à 4 moments différents entre 52 à 76 heures après le stade $95 \%$ d'éclos) et (ii) en utilisant deux rations alimentaires (différentes d'un facteur 3 ).

Les résultats montrent que les débits d'eau et d'air influencent fortement la mortalité : une élévation de $25 \%$ du débit d'eau et un faible débit d'air ont des effets négatifs durant la période d'alimentation mixte (jours 3-4). Le sens de circulation de l'eau influence la mortalité durant toute la période d'élevage concernée. Un courant ascendant améliore la survie. Ces résultats ont été confortés lorsque des changements de sens de circulation de l'eau ont été pratiqués en cours d'élevage. Ils modifient quasi immédiatement (dans les 8-24 heures) l'évolution des mortalités observées : le passage d'un courant ascendant à un courant descendant entraîne de fortes mortalités et le changement inverse se traduit par une diminution, voire un arrêt de la mortalité. Les conditions d'alimentation testées n'influencent pas la survie larvaire ; elles n'interagissent pas non plus avec le sens de circulation de l'eau sur la survie.

Par ailleurs, ni les facteurs influençant l'hydrodynamique dans les bassins, ni le décalage de la première alimentation n'ont d'effet sur la croissance larvaire. Seule l'augmentation de la quantité de proies distribuées permet une augmentation significative de la croissance.

En conclusion, l'hydrodynamique dans les bassins a une forte influence sur les survies des larves. Ceci impose, d'un point de vue pratique, un contrôle précis des débits d'eau et d'air. La disponibilité en proies et leur accessibilité pour les larves ne semblent pas devoir être liées aux effets des facteurs hydrodynamiques.

Mots-clés : turbot, techniques d'élevage, alimentation.

\section{HYDRODYNAMICS EFFECTS IN LARVAE REARING TANKS ON SURVIVAL AND GROWTH OF TURBOT (PSETTA MAXIMA).}

\section{ABSTRACT}

During larval rearing phase of turbot, a substantial variability in survival and growth is frequently observed. In this paper, we studied the effects of hydrodynamics in larvae rearing tanks on the survival and the growth of fed and starved larvae, using the following conditions : 
- water flows of 600 and $1200 \mathrm{ml} \cdot \mathrm{mn}^{-1}$, constant and progressive ones,

- air flows of 10 and $30 \mathrm{ml} \cdot \mathrm{mn}^{-1}$,

- downward and upward water circulation, with direction change during the rearing phase.

We also studied the interactions between water circulation direction and feeding conditions by (1) shifting the first feeding day ( 4 occasions, at 8 hours intervals, from 52 to 76 hours after $95 \%$ of hatching) and (2) using 2 amounts of preys (which differ by a factor 3 ).

The results show that water and air flows have a strong effect on the mortality : a $25 \%$ water flow increase and a low air flow have negative effects during the mixed feeding period. The water circulation direction influenced strongly the mortality during the whole period : an upward water direction leads to the best survival. These results were confirmed under water circulation direction changes. Under these conditions, the mortality was immediatly modified the change from an upward to a downward direction provides high mortalities and conversely. The feeding conditions have no effects on the larvae survival. There is no interactions with water circulation direction on survival.

During this rearing phase, nor hydrodynamics conditions, nor the first feeding day shift have an effect on the growth. The amount of preys is the only condition which provides a significant effect on the larval growth.

In conclusion, hydrodynamics in rearing tanks has a strong effect on the larvae survival. In order to limit the variability of turbot rearing performances, the control of the air and water flows is of prime importance. The prey avalaibility and their accessibility for the larvae do not seem to be linked with the hydrodynamics effects.

Key-words : turbot, rearing methods, feeding.

\section{INTRODUCTION}

Durant la phase d'élevage larvaire du turbot, une grande variabilité des performances de survie et de croissance est généralement observée, y compris entre différents bassins d'un même lot de larves (MINKOFF et BROADHURST, 1994 ; STOTTRUP, 1993). Cette variabilité peut nuire à l'interprétation de résultats expérimentaux. De nombreuses hypothèses ont été avancées pour expliquer ces résultats : qualité d'eau, qualité des oeufs, conditions d'élevage, aspects alimentaires et nutritionnels, environnement microbien (PERSON-LE RUYET, 1989 ; GATESOUPE, 1990 ; BERGH et al., 1991 ; STOTTRUP, 1993 ; LECLERCQ, 1994 ; MINKOFF et BROADHURST, 1994). Parmi celles-ci, certaines (effet site, qualité des oeufs) sont susceptibles d'expliquer des différences de performances entre différentes bandes élevées dans des conditions différentes. Elles expliquent rarement les différences de performances enregistrées au sein d'un même lot élevé dans plusieurs bassins dans des conditions a priori identiques. Les aspects nutritionnels et microbiens ont été particulièrement étudiés par de nombreux auteurs. Par contre, si les conditions d'élevage ont été étudiées initialement pour définir les techniques de base de l'élevage, depuis lors peu de travaux ont été effectués sur l'influence de l'hydrodynamique dans les bassins d'élevage larvaire (KILLGORE et al., 1987 ; CHESNEY, 1989). D'une manière générale, l'eau est utilisée principalement pour maintenir la qualité du milieu d'élevage (CRIPPS et POXTON, 1992). En élevage larvaire, les débits sont trop faibles pour que le courant résultant soit suffisant pour assurer un auto-nettoyage des bassins. Les méthodes d'élevage dites " en eau claire " utilisent le courant d'eau combiné à une aération pour accroître le taux de rencontre entre les proies et les larves et, ainsi, optimiser l'alimentation des larves (PERSON-LE RUYET, 1989 ; CHESNEY, 1989 ; GULBRANDSEN, 1991, 1993 ; VAN DER MEEREN, 1991 ; HERNANDEZ-CRUZ et al., 1994). Par contre, les méthodes dites « en eau verte " sont basées sur l'absence de renouvellement de l'eau avec ou sans 
aération (TANDLER et HELPS, 1985 ; STOTTRUP, 1993). En définitive, il est aujourd'hui difficile de cerner l'influence, sur les performances larvaires, des facteurs caractérisant l'hydrodynamique dans les bassins.

Durant la phase d'élevage concernée, on passe successivement par trois périodes caractérisées par différentes sources d'énergie alimentaire utilisées par les larves : une période d'alimentation endogène, une période d'alimentation mixte lorsque les larves commencent à s'alimenter à partir d'une source extérieure et une période avec une alimentation uniquement exogène. Le passage à une alimentation exogène est considérée comme la période critique de l'élevage et est reconnu comme étant une des causes majeures de mortalité des larves (KAMLER, 1992). Aussi, tous les facteurs qui peuvent influencer la rencontre entre les larves et leurs proies sont importants à considérer. L'hydrodynamique dans les bassins est un de ceux-là (PERSON-LE RUYET, 1989 ; SKRESLET, 1989 ; GULBRANDSEN, 1993 ; VAN DER MEEREN, 1991).

Dans cet article, nous aborderons successivement un ensemble de facteurs qui peuvent modifier l'hydrodynamique dans les bassins d'élevage larvaire de turbot. L'influence du débit d'eau, du débit d'air et du sens de circulation de l'eau dans les bassins (ascendant ou descendant) sera étudiée sur la survie et la croissance des larves jusqu'au jour 10 (J10). L'effet de variations des débits d'eau et d'inversion du sens de circulation de l'eau en cours d'élevage sera également abordé. Afin de cerner si les effets des facteurs étudiés sont liés à la capacité des larves à se nourrir, des expériences complémentaires ont été menées. Les trois facteurs étudiés (débit d'air, d'eau et sens de circulation de l'eau) ont été appréhendés en condition à jeun. Les effets du sens de circulation de l'eau dans les bassins ont été étudiés dans différentes conditions d'alimentation des larves, en décalant la première distribution de proies ainsi qu'en modifiant la quantité de proies distribuées.

\section{MATÉRIEL ET MÉTHODES}

\subsection{Conditions générales}

\section{Larves utilisées}

Les larves utilisées sont issues de géniteurs captifs, provenant de plusieurs saisons de reproduction, et élevés à l'IFREMER, centre de Brest. Pour toutes les expériences (excepté l'expérience 6), le Jour 0 (J0) est le jour d'éclosion. Sur les échelles horaires, l'Heure $0(\mathrm{HO})$ correspond à $8 \mathrm{~h}$ du matin au jour 1 (J1) (sauf expérience 6), heure à laquelle les larves sont mises dans les bassins d'élevage larvaire (sauf expérience 5).

\section{Méthodes générales d'élevage}

Les larves sont élevées selon une méthode " en eau claire ". Elles sont mises à J1 dans des bassins cylindro-coniques de $150 \mathrm{~L}$ décrits par PERSON-LE RUYET (1989), avec une arrivée d'eau à la surface de l'eau, une évacuation à mi-hauteur et une aération. La densité initiale est de 20-30 larves par litre. L'intensité lumineuse est de 80-100 lux de J1 à J2 puis de 1000 lux à partir de J3. Afin d'éviter les interactions des facteurs étudiés et contrôlés avec la photopériode, la durée d'éclairement est de $24 \mathrm{~h} / \mathrm{jour}$. La température est augmentée progressivement de $13^{\circ} \mathrm{C}$ à $\mathrm{J} 1$ (température d'incubation) à $18^{\circ} \mathrm{C}$ à $\mathrm{J} 6$, puis est maintenue constante.

Les larves sont nourries avec des rotifères (Brachyonus plicatilis) de J3 (Première Alimentation, PA) à la fin des expériences. Les rotifères sont élevés en eau de mer et alimentés avec des algues (Platymonas suecica), de la levure de boulanger, de l'huile de foie de morue et un premix vitaminique. Avant d'être distribués, ils sont enrichis avec de la levure de bière et de l'huile de foie de morue, et sont distribués en continu aux larves durant 
$20 \mathrm{~h} / \mathrm{jour}$, les $4 \mathrm{~h}$ restantes/jour étant mises à profit pour assurer la maintenance et le suivi des élevages. La quantité de proies distribuées est basée sur le nombre initial de larves à raison de : $50,70,100,120,140,150,160$ rotifères/larve respectivement de $\mathrm{J} 3$ à $\mathrm{J} 10$ (sauf expérience 6).

\section{Contrôle des paramètres}

Dans toutes les expériences, le débit de l'eau de chacun des bassins est contrôlé deux fois par jour à l'aide de débitmètres électroniques à effet Hall associés à deux compteurs à impulsion avec affichage par scrutation des valeurs. La variation maximale entre deux contrôles a été de $10 \mathrm{ml} \cdot \mathrm{mn}^{-1}$. Des débitmètres de type Aalborg sont utilisés pour contrôler le débit de l'air deux fois par jour.

Lors de la distribution des larves dans les bassins, trois bassins "perdus" sont positionnés aléatoirement dans le dispositif expérimental afin d'être comptés exhaustivement pour avoir une bonne estimation du nombre initial de larves de chacun des bacs. Les larves mortes sont recueillies par purge et dénombrées deux fois (ou quatre dans l'expérience 6) par jour lorsque les larves sont alimentées, et trois ou quatre fois par jour pour les larves laissées à jeun. Le nombre de larves est compté exhaustivement en fin d'expérience.

Les incertitudes liées à la petite taille des larves, des contraintes liées à certains systèmes hydrodynamiques utilisés et notre expérience des expérimentations sur la phase larvaire nous ont amenés à considérer que :

- les effectifs initiaux et finaux, dénombrés, de larves vivantes sont fiables et nous autorisent à calculer des taux de survie en fin d'expérience ; les taux de survie ayant, de plus, davantage d'intérêt pratique que le taux de mortalité ;

- le taux de mortalité calculé sur la base du dénombrement des morts régulièrement recueillis (cf., en annexe, le mode de calcul) était le plus à même de donner une image de la dynamique de la mortalité.

En conséquence, le taux de survie est utilisé pour comparer les effets des facteurs un jour donné. Le taux de mortalité est utilisé pour comparer les résultats sur une période donnée.

Vingt-cinq larves sont prélevées régulièrement, fixées dans du formol à l'eau de mer à $5 \%$ pendant au moins deux semaines avant d'être pesées individuellement au $\mu \mathrm{g}$ près (poids humide).

\subsection{Plans d'expériences}

\subsubsection{Effets du débit d'eau (expériences 1 et 2)}

Les effets sur la mortalité et la croissance de trois débits d'eau sont étudiés : 600 et $1200 \mathrm{ml} \cdot \mathrm{mn}^{-1}$ à un niveau constant durant toute l'expérience et un débit progressif : $600 \mathrm{ml} \cdot \mathrm{mn}^{-1}$ de $\mathrm{J} 1$ à $\mathrm{J} 3$ puis une augmentation régulière jusqu'à $1200 \mathrm{ml}^{\mathrm{m}} \mathrm{mn}^{-1}$ à $\mathrm{J} 7$ (condition 600-1200). Le débit d'air est de $10 \mathrm{ml} \mathrm{mn}^{-1}$ dans tous les bassins et l'expérience dure 7 jours. Les larves sont échantillonnées à $\mathrm{J} 1, \mathrm{~J} 3$ et $\mathrm{J} 7$. Trois réplicats sont mis en oeuvre.

Dans l'expérience 2, les larves sont à jeun, 2 débits d'eau : 600 et $1400 \mathrm{ml}^{\mathrm{m} \mathrm{m}^{-1}}$, avec quatre réplicats par condition sont utilisés. 


\subsubsection{Effets du débit d'air (expériences 3, 3bis et 4)}

L'effet de trois débits d'air est étudié lors d'une expérience répliquée (exp. 3 et 3 bis) avec deux débits d'air constants $\left(10\right.$ et $30 \mathrm{ml} \cdot \mathrm{mn}^{-1}$ ) et un débit variable (accru de 10 à

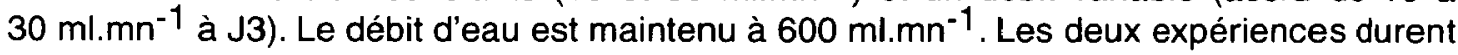
respectivement 10 et 9 jours et chaque traitement est répliqué 6 et 3 fois, respectivement pour les expériences 3 et 3bis. Dans l'expérience 3bis, 28 prélèvements de $10 \mathrm{ml}$ d'eau ont été effectués dans la totalité du volume occupé par les larves de chacun des bassins afin d'étudier la répartition des proies. La mortalité de larves à jeun est étudiée dans les mêmes conditions de débit, avec 3 réplicats lors de l'expérience 4 .

\subsubsection{Etude du sens de circulation de l'eau (expériences 5 et 5 bis)}

Lors de 2 expériences, deux systèmes d'élevage larvaire, dont le sens de circulation de l'eau dans les bassins est opposé, ont été comparés:

- un système " Hydrodynamique Descendante " (HD) décrit par PERSON-LE RUYET (1989) avec l'arrivée d'eau située au-dessus de la surface de l'eau, la sortie située à $40 \mathrm{~cm}$ de profondeur, au-dessus de la partie conique du bassin. Le débit d'eau est de $600 \mathrm{ml} . \mathrm{mn}^{1}$; le débit d'air de $10 \mathrm{ml} \cdot \mathrm{mn}^{-1}$;

- un système "Hydrodynamique Ascendante» (HA) ayant un sens de circulation opposé au précédent et conçu afin d'éviter des volumes morts : l'arrivée d'eau est située au fond du bassin et la sortie à la surface, il n'y a pas d'aération. Le débit d'eau, plus élevé, est de $1000 \mathrm{ml} \cdot \mathrm{mn}^{-1}$.

Pour les deux systèmes, la surface du tamis d'évacuation, la taille des mailles (180 $\mu$ ) de ce tamis sont identiques.

De plus, lors des expériences 5 et 5 bis, ont été étudiés :

- l'effet du système hydrodynamique mis en oeuvre durant l'incubation (phase précédant la phase d'élevage larvaire proprement dite) en utilisant :

* des incubateurs dont l'arrivée d'eau est au fond, avec une aération, pour créer une circulation d'eau ascendante ;

* des incubateurs dont l'arrivée d'eau est en surface afin d'obtenir un courant descendant, l'aération étant supprimée ;

- l'effet d'un changement de sens de circulation de l'eau dans les bassins pendant la phase d'élevage larvaire : dans chacune des deux expériences, le sens de circulation de l'eau dans les bassins a été inversé une fois.

Trois réplicats ont été mis en oeuvre pour chacune de ces deux expériences qui durent 8 jours.

\subsubsection{Relations entre hydrodynamique et alimentation (expérience 6)}

Afin d'analyser les interactions possibles entre l'hydrodynamique des bassins et les conditions d'alimentation, un plan expérimental à trois facteurs croisés, sans répétition, a été utilisé. Les facteurs étudiés sont :

- d'une part, l'hydrodynamique des bassins avec 2 niveaux constitués par les deux systèmes décrits précédemment (HA et HD) ; 
- d'autre part, (1) le Décalage de la Première Alimentation (DPA) avec 4 niveaux constitués de 4 moments différents de première distribution d'aliment, toutes les 8 heures de $\mathrm{H} 52$ à $\mathrm{H} 76$ (H0 étant définie par l'heure à laquelle $95 \%$ des larves sont écloses), et (2) la Quantité de Proies Distribuées (QPD) : deux schémas alimentaires (QPD1 et QPD2) ont été utilisés (tableau I). Ils ont été décalés toutes les 8 heures afin de prendre en compte le décalage de la première distribution d'aliment. Les rotifères ont été distribués en continu pendant 20 heures.

\section{Tableau I}

Relations entre hydrodynamique et alimentation des larves : schémas alimentaires utilisés (QPD : Quantité de Proies Distribuées) en nombre de rotifères pour chaque période successive de $24 \mathrm{~h}$ et par larve initiale.

\section{Table I}

Relation between tank hydrodynamics and feeding : feeding schemes (QPD) used, expressed as rotifers numbers per initial larvae for each successive $\mathbf{2 4}$ h-period.

\begin{tabular}{|c|c|c|c|c|c|}
\hline pour les périodes, en heure & $0-24$ & $24-48$ & $48-72$ & $72-96$ & $96-120$ \\
\hline QPD 1 (rotifères/larve) & 40 & 70 & 100 & 120 & 140 \\
\hline QPD 2 (rotifères/larve) & 120 & 210 & 300 & 360 & 280 \\
\hline
\end{tabular}

Pour cette expérience qui a duré sept jours, les larves pesaient $265 \mu \mathrm{g}$ à $\mathrm{HO}$. Elles ont été transférées dans les bacs d'élevage cylindro-coniques de $150 \mathrm{~L}$ à J1. La densité initiale est de 15 larves/l. Les autres conditions d'élevage sont identiques aux autres expériences.

\subsection{Traitement des données}

Pour les expériences 1 à 5 bis, les survies et mortalité sont analysées, après transformation angulaire (arcsinus $\sqrt{ } \mathrm{x}$ ) et vérification de l'homogénéité des variances par un test de Bartlett, à l'aide d'une ANOVA à un facteur, suivi d'un test de Tuckey en cas de différence significative. Les poids des larves sont comparés à l'aide d'une ANOVA hiérarchisée afin de tenir compte de l'effet bassin. Pour l'expérience 6, une ANOVA à 3 facteurs est utilisée.

\section{RÉSULTATS}

\subsection{Effet du débit d'eau}

Aucune différence de mortalité selon les débits d'eau n'a été enregistrée ( $P=0,115)$ durant les deux premiers jours, c'est-à-dire jusqu'à la première distribution d'aliment (fig. 1). Après J3, la mortalité augmente rapidement dans toutes les conditions. L'augmentation de mortalité de J3 au matin ( $\mathrm{H} 48$ ) jusqu'à J4 après-midi $(\mathrm{H} 80)$ est significativement supérieure dans la condition $600-1200 \mathrm{ml} \cdot \mathrm{mn}^{-1}(59,2 \%, P=0,018)$ que dans les conditions 600 et $1200 \mathrm{ml} \cdot \mathrm{mn}^{-1}$ (43,4 et 35,5\% respectivement). De J4 (H80) à J7, le taux de mortalité n'est pas significativement différent entre les différentes conditions $(P=0,437)$. Les survies finales à $J 7$ sont différentes $(P=0,0113)$, la survie dans la condition $600-1200$ est significativement inférieure à celle obtenue dans la condition 1200. 
Les poids moyens à $\mathrm{J} 3(193 \pm 1,5 ; 196 \pm 1,4 ; 185 \pm 4,8 \mu \mathrm{g}$ respectivement pour les conditions $600 ; 600-1200 ; 1200 ; P=0,116)$ ainsi que les taux de croissance pondérale de J3 à J7 ( $P=0,876)$ sont identiques quelles que soient les conditions. Le poids moyen final des différents lots $(278 \pm 32 ; 279 \pm 46 ; 242 \pm 25 \mu \mathrm{g}$ respectivement pour les conditions 600 ; $600-1200 ; 1200)$ est identique ( $P=0,802)$.

Les taux de mortalité des larves à jeun sont identiques dans les conditions 600 et 1400 jusqu'à J3 (H48) (expérience 2, fig. 2). Ensuite, dans la condition 1400, la mortalité augmente rapidement et conduit à des différences significatives de survie entre les conditions à $H 64(P=0,044)$. Cette différence disparaît lorsque les larves atteignent $\mathrm{J} 7$, la totalité des larves mourant avant la $158^{\text {eme }}$ heure.

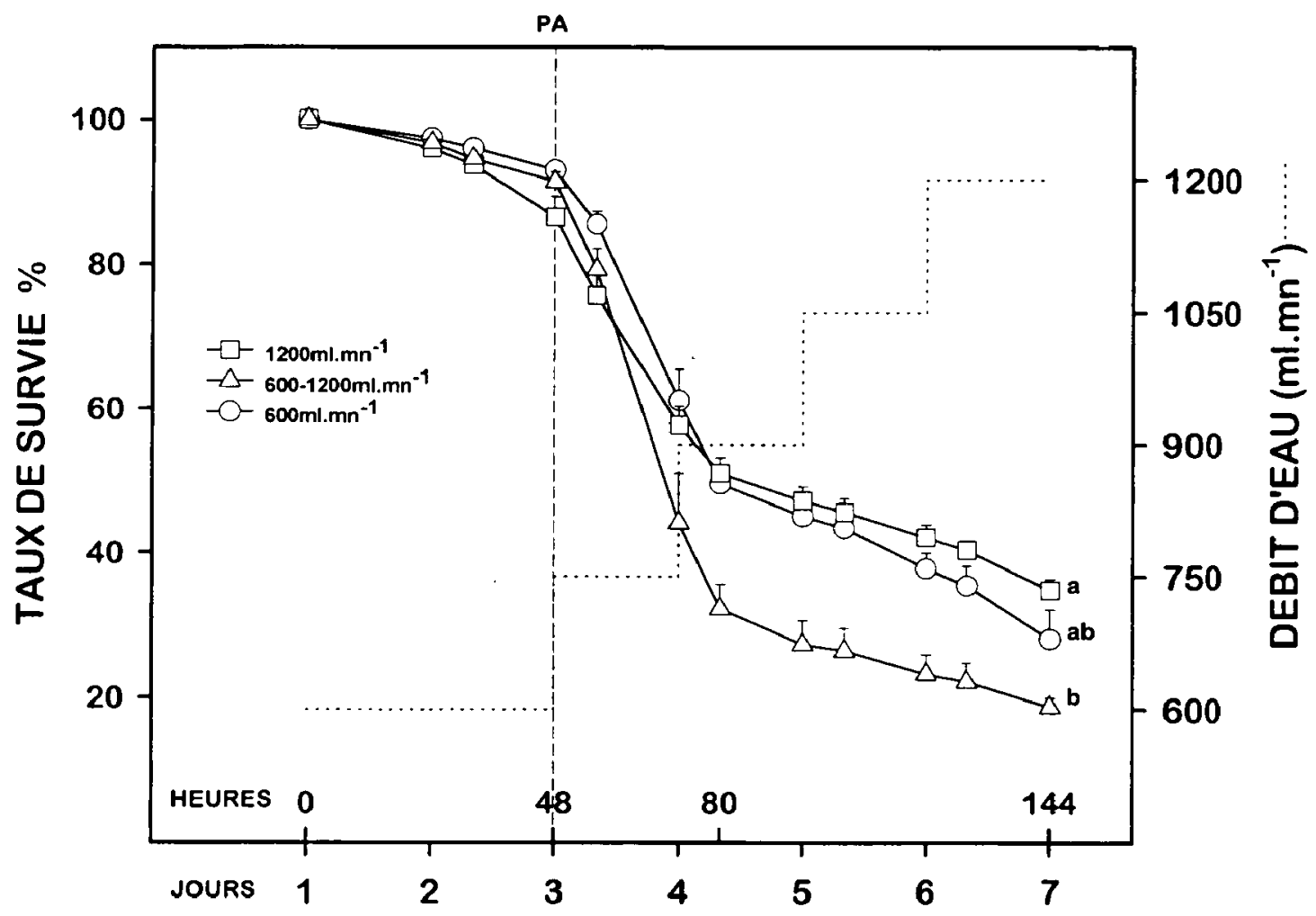

Figure 1

Expérience 1 : Taux de survie (\%) de larves nourries en fonction du débit d'eau. La ligne pointillée représente l'évolution des débits d'eau pour la condition 600-1200. PA indique le moment de la première distribution alimentaire. J0 : jour de l'éclosion. $\mathrm{HO}: 8 \mathrm{~h}$ à J1. Les erreurs standards sont indiquées. Des lettres minuscules identiques indiquent que les moyennes ne sont pas significativement différentes.

\section{Figure 1}

Experiment 1 : Survival rates (\%) of fed turbot larvae in function of water flow. The dotted line represents the change of water flow under the condition 600-1200. PA : first feeding. DO = hatching. $\mathrm{HO}: 08.00$ a.m. at D1. The standard errors are given. Any common letter indicates no significant difference. 


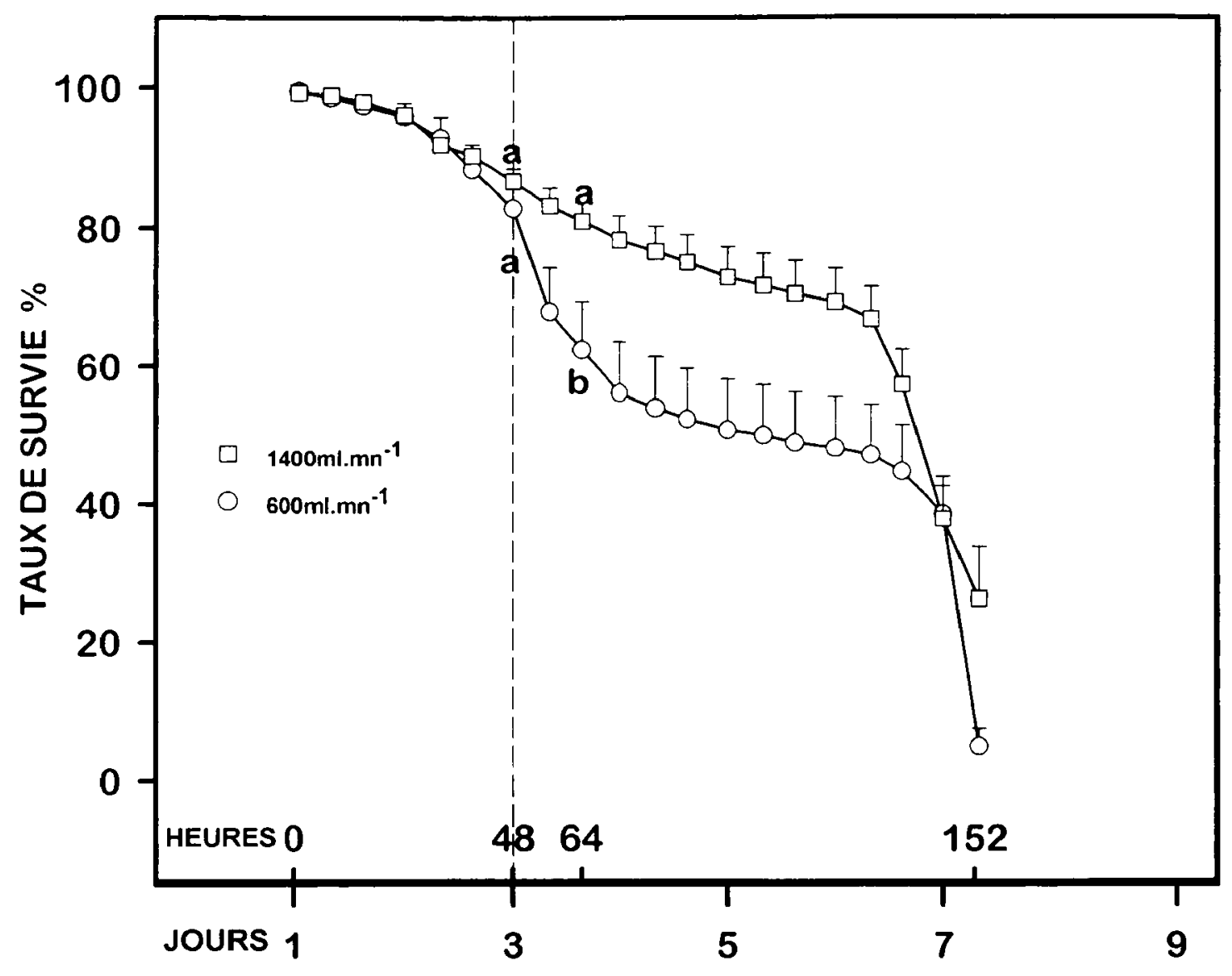

Figure 2

Expérience 2 : Survie (\%) de larves non alimentées en fonction du débit d'eau. La ligne tiretée indique le jour 3 , date habituelle de première distribution alimentaire. Les erreurs standards sont indiquées. Des lettres minuscules identiques indiquent que les moyennes ne sont pas significativement différentes.

Figure 2

Experiment 2 : Survival rates (\%) of starved turbot larvae in function of water flow. DO = hatching. HO : 08.00 a.m. at D1. The broken line indicates D3, when the larvae are usually fed. The standard errors are given. Any common letter indicates no significant difference.

\subsection{Effet du débit d'air}

II n'y a pas d'effet du débit d'air sur la mortalité des larves jusqu'à J3 (fig. 3A) et les résultats sont identiques pour les 2 expériences. Par contre, entre $\mathrm{J} 3$ et $\mathrm{J} 5$, le taux de mortalité avec un débit d'air de $10 \mathrm{ml} \cdot \mathrm{mn}^{-1}$ est supérieur à celui obtenu dans les deux autres conditions ( $P=0,0001$ et $P=0,006$, respectivement pour les deux expériences). Après $J 5$, les taux de mortalité ne sont pas différents entre les conditions $(P=0,107$ et $P=0,462$, respectivement pour les deux expériences). Les taux de survie à la fin des expériences sont plus bas dans la condition $10 \mathrm{ml} . \mathrm{mn}^{-1}$ que dans les deux autres conditions (tableau II). 


\section{Tableau II}

Effets du débit d'air : taux de survie en fin d'expérience (J10 et J8, respectivement pour les expériences 3 et 3 bis) pour des débits d'air de 10 et $30 \mathrm{ml}^{\mathrm{m}} \mathrm{mn}^{-1}$ constants et un débit d'air variable (accru de 10 à $30 \mathrm{ml}^{\mathrm{m} n^{-1}}$ à J3). Des lettres minuscules identiques indiquent que les moyennes ne sont pas significativement différentes.

\section{Table II}

Air flow effects : survival rates at the end of the experiments (D10 and D8 for experiments 3 and 3 bis respectively) for constant 10 and $30 \mathrm{ml} . \mathrm{mn}^{-1}$ air flows and an increased flow from 10 to $30 \mathrm{ml} \cdot \mathrm{mn}^{-1}$ at D3. Any common letter indicates no significant difference.

\begin{tabular}{|c|c|c|c|c|}
\hline débit d'air $\left(\mathrm{ml}^{\prime} \mathrm{mn}^{-1}\right)$ & 10 & $10-30$ & 30 & Probabilité \\
\hline expérience 3 & $24.3 \pm 3.1 \mathrm{a}$ & $64.3 \pm 3.6 \mathrm{c}$ & $47.1 \pm 3.1 \mathrm{~b}$ & $<0.0001$ \\
\hline expérience 3bis & $17.9 \pm 3.2 \mathrm{a}$ & $30.9 \pm 2.5 \mathrm{~b}$ & $41.5 \pm 2.9 \mathrm{~b}$ & 0.014 \\
\hline
\end{tabular}

Dans l'expérience 3bis, la densité moyenne des proies dans les bassins est comprise entre 0,23 à $0,66 \mathrm{proie} / \mathrm{ml}$ et semble indépendante du débit d'air (fig. 3B).

A tout moment, le poids moyen des larves est identique pour toutes les conditions $(P=0,835,0,205$ et 0,127 respectivement à $\mathrm{J} 3, \mathrm{~J} 5$ et $\mathrm{J} 7$ pour l'expérience $3 ; P=0,166,0,127$ et 0,059 respectivement à $\mathrm{J} 5, \mathrm{~J} 7$ et $\mathrm{J} 9$ pour l'expérience $3 \mathrm{bis}$ ). La croissance est nulle jusqu'à $\mathrm{J} 5$, bien que les larves aient été nourries (fig. $3 \mathrm{C}$ ).

La mortalité des larves maintenues à jeun augmente entre J3 et J5 (expérience 4) de la même manière que lorsque les larves sont alimentées (fig. 4), mais il n'existe pas de différence entre les conditions ( $P=0,068)$.

\subsection{Sens de circulation du courant d'eau}

Un effet rapide du sens de circulation de l'eau sur la mortalité est observé (expérience 5, fig. 5) : 8 heures après la fin de l'éclosion, la mortalité est supérieure dans le système $H D$, en circulation descendante, comparée à celle obtenue dans le système ascendant $(P=0,0201)$. Une inversion du sens de circulation à $J 3$, lors de la première alimentation, conduit à une modification de l'évolution de la mortalité : elle s'arrête soudainement lors du passage d'un courant descendant (HD) à un courant ascendant (HA) (après 8 heures, les taux de mortalité sont significativement différents : $P=0,0004$ ), et augmente lors du changement de HA à $H D(P=0,0448,24$ heures après le changement). $A$ la fin de l'expérience (J6), le taux de survie est supérieur lorsque les larves sont élevées dans un courant ascendant durant la période J3-J6, quel que soit le système utilisé avant J3 $(P=0,0001)$.

Lors de l'expérience 5 bis, aucune différence de mortalité n'existe entre les conditions à la fin de l'éclosion $(\mathrm{HO})$, quel que soit le système utilisé durant l'incubation. La mortalité évolue ensuite différemment en fonction des conditions hydrodynamiques présentes après $H 0(P=0,0013$ à $H 24)$, indépendamment des conditions ayant prévalu lors de l'incubation (fig. 5B) : les larves élevées dans un système ascendant ont un taux de survie supérieur à celles élevées dans un courant d'eau descendant $(P=0,0201)$. Cette différence s'accroît régulièrement. Lorsqu'un changement du sens de circulation de l'eau est pratiqué à J5, ceci conduit à une modification de la cinétique de la mortalité : la mortalité s'arrête quasi instantanément lors d'un passage de $H D$ à $H A$ ( $P=0,0422$ après 8 heures) et augmente après le passage de HA à $H D(P=0,0047,48$ heures après le changement). A la fin de l'expérience (J9), les taux de survie sont différents $(P=0,0001)$ : la survie est plus élevée si les larves sont élevées dans un système ascendant (HA) après $\mathrm{J} 3$. 

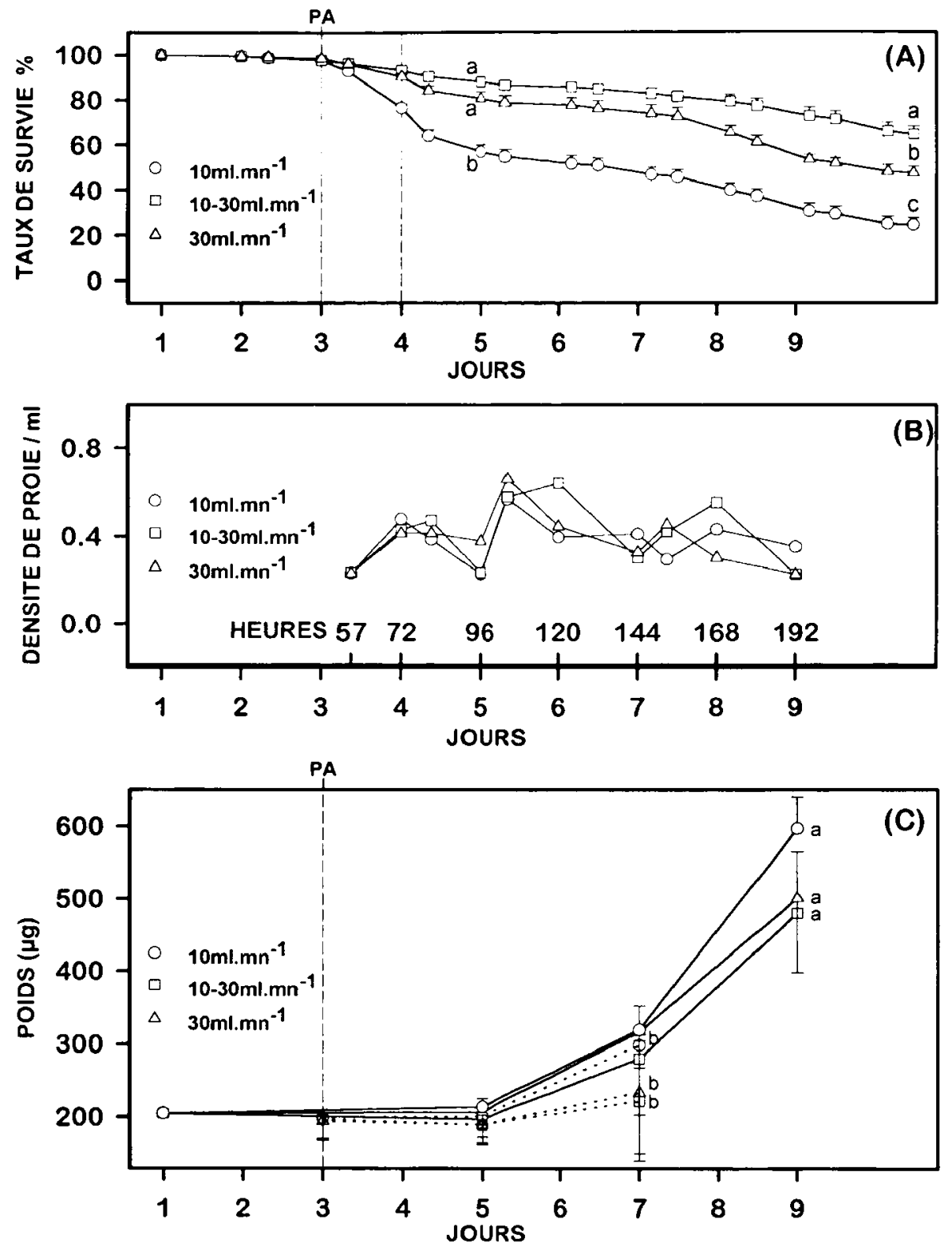

Figure 3

Expériences 3 et 3 bis : Taux de survie (\%) (A) (pour l'expérience 3, voir les taux de survie Tableau II) et poids (C) (expérience 3 : ligne continue, expérience 3 bis : ligne pointillée) $(\mu \mathrm{g})$ de larves de turbot alimentées en fonction du débit d'air et densités moyennes de proies dans les bassins (en nombre de proies/ml) (B). Les lignes tiretées délimitent la période J3-J4. PA indique le moment de la première distribution alimentaire. J0 : jour de l'éclosion. $\mathrm{HO}: 8 \mathrm{~h}$ à $\mathrm{J} 1$. Les erreurs standards sont indiquées. Des lettres minuscules identiques indiquent que les moyennes ne sont pas significativement différentes.

Figure 3

Experiments 3 and 3 bis : Survival rates (\%) (A) (experiment 3, see the survival rates on Table II) and weights (C) (experiment 3 : continuous lines, and experiment 3bis : dotted lines) $(\mu \mathrm{g})$ of fed turbot larvae in function of air flow and mean prey density (prey number/mI) (B). The broken lines delimit D3-D4 period. PA : first feeding. $\mathrm{DO}=$ hatching. $\mathrm{HO}: 08.00 \mathrm{a} . \mathrm{m}$. at D1. The standard errors are given. 


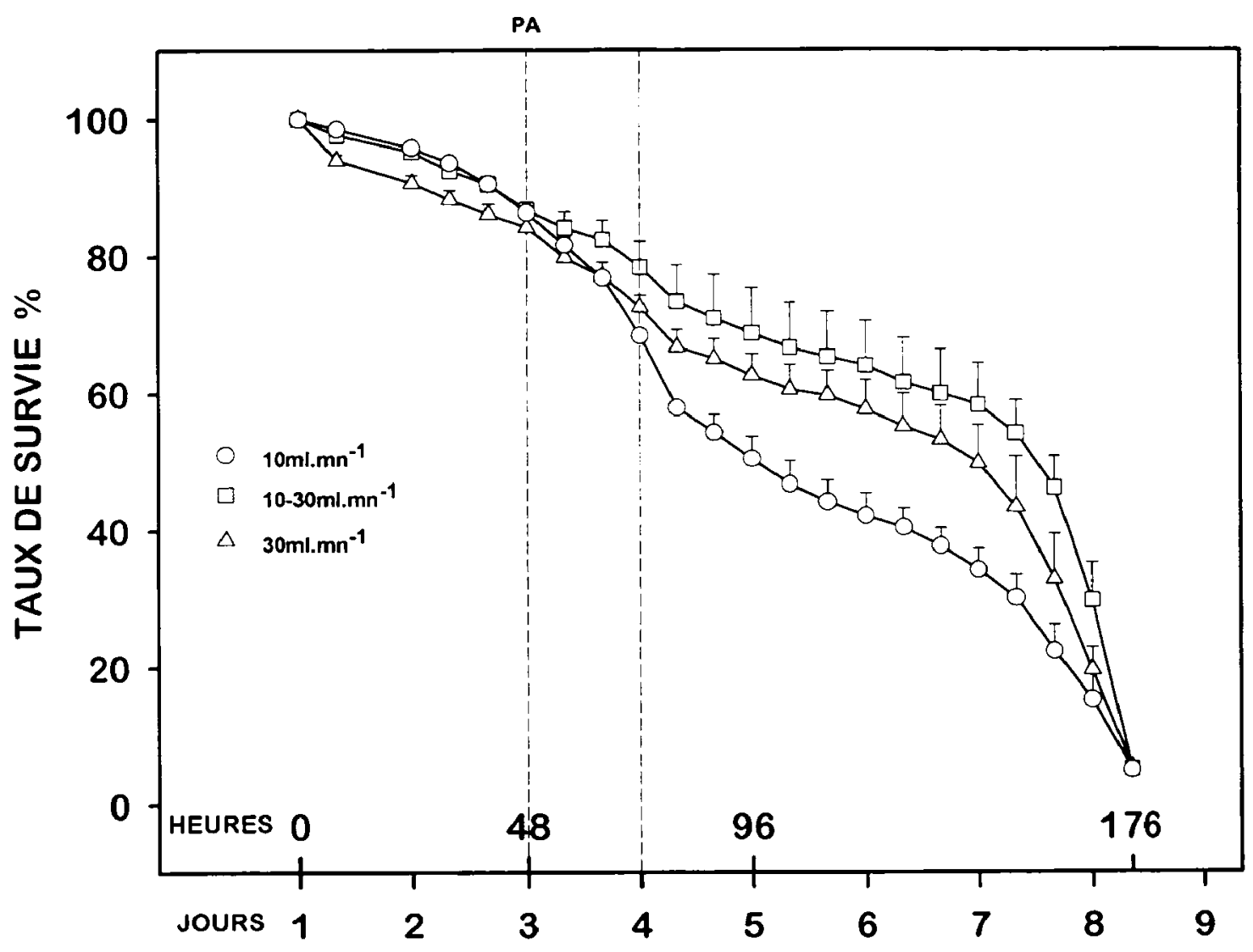

Figure 4

Expérience 4 : Survie de larves non alimentées en fonction du débit d'air. Les lignes tiretées délimitent la période J3-J4. PA indique la date habituelle de la première distribution alimentaire. J0 : jour de l'éclosion. $\mathrm{HO}: 8 \mathrm{~h}$ à J1. Les erreurs standards sont indiquées.

\section{Figure 4}

Experiment 4 : Survival rates of starved turbot larvae in function of air flow. The broken lines delimit D3-D4 period. PA : time when the larvae are usually fed. $\mathrm{DO}=$ hatching. $\mathrm{HO}: 08.00 \mathrm{a} . \mathrm{m}$. at D1. The standard errors are given.

\subsection{Relations entre hydrodynamique et alimentation}

Le seul facteur qui affecte la mortalité jusqu'à J2 et durant la période J3-J4 (tableau III), et en conséquence à la fin de l'expérience (tableau IV), est l'hydrodynamique des bassins. Le taux de mortalité enregistré dans le système HD est supérieur à celui enregistré dans le système HA. Ni le décalage de l'alimentation, ni les différents schémas alimentaires pratiqués n'ont d'influence sur la mortalité.

À J3, le poids des larves (tableau $\mathrm{V}$ ) ne dépend que de l'hydrodynamique des bassins : la diminution de poids enregistrée dans le système HD est supérieure à celle enregistrée dans le système HA. La croissance durant la période J3-J5 est indépendante des facteurs étudiés. À J5, le poids des larves ne dépend que de la quantité de proies distribuées : plus la quantité distribuée est importante, plus le poids obtenu est élevé. De $\mathrm{J} 3 / 5$ à $\mathrm{J} 7$, un effet du rationnement est également observé mais, à $\mathrm{J} 7$, il n'y a plus aucune relation entre le poids des larves et les facteurs étudiés. 

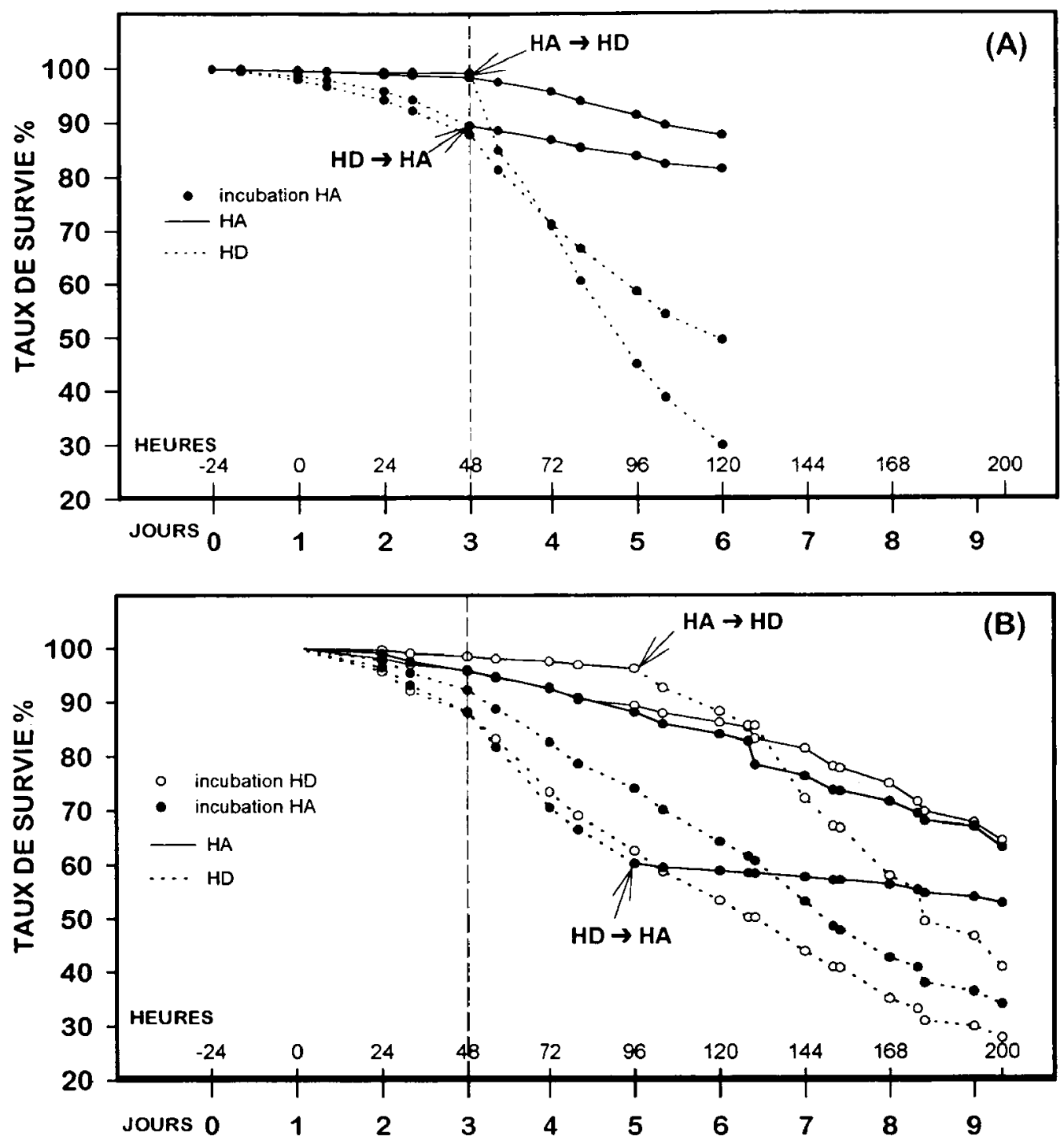

Figure 5

Taux de survie de larves de turbot alimentées en fonction du sens de circulation de l'eau (ascendant/descendant) dans les bassins. (A) : expérience 5 ; (B) : expérience 5 bis. Les courbes de survie démarrent à la mise en bassin d'élevage larvaire : juste avant l'éclosion pour l'expérience 5, à la fin de l'éclosion pour l'expérience 5 bis. Chaque condition est caractérisée par un changement de sens de circulation de l'eau dans les bassins (HA $\rightarrow$ HD ou HD $\rightarrow$ HA). Ligne continue : sens de circulation de l'eau ascendant et ligne pointillée : sens descendant. J0 : jour de l'éclosion. HO : $8 \mathrm{~h}$ à $\mathrm{J1}$. La ligne tiretée indique la date de première distribution alimentaire.

Figure 5

Survival rates of fed turbot larvae in function of the water circulation direction (upward or downward) in the tanks. (A) : experiment 5 ; (B) : experiment 5 bis. The curves begin at the moment when larvae are placed in larvae rearing tanks : just before hatching in experiment 5 , at the end of hatching in experiment 5bis. Each condition is characterized by a change of water circulation direction (HA $\rightarrow$ HD or $H D \rightarrow H A)$. Continuous line : upward water circulation direction, dotted line : downward direction. $\mathrm{DO}=$ hatching. $\mathrm{H0}: 08.00 \mathrm{a} . \mathrm{m}$. at D1. The broken line indicates first feeding. 
Tableau III

Effets de l'Hydrodynamique dans les bassins (HA et HD), des Quantités de proies distribuées (QPD1 et QPD2) et du Décalage de la Première Distribution alimentaire (DPA) (de +52 à +76 heures après que $95 \%$ des larves soient écloses) sur le taux de mortalité (\%) de larves de turbot jusqu'à J2, durant les périodes $\mathrm{J3}-\mathrm{J} 4$ et $\mathrm{J} 5-\mathrm{J} 7$ (tableau du haut) avec résultats de l'analyse de variance (tableau du bas). Les chiffres en caractères gras indiquent les effets significatifs.

Table III

Effects of tank hydrodynamics (HA or HD), feeding scheme (QPD1 or QPD2) and first feeding shift (DPA) (from +52 hours to +76 hours after $95 \%$ of the larvae hatched) on mortality rate (\%) of turbot larvae up to $\mathrm{J} 2$, during $\mathrm{J} 3-\mathrm{J} 4$ period and J5-J7 one with 3way ANOVA results. Heavy types pointed out factors which have significant effects.

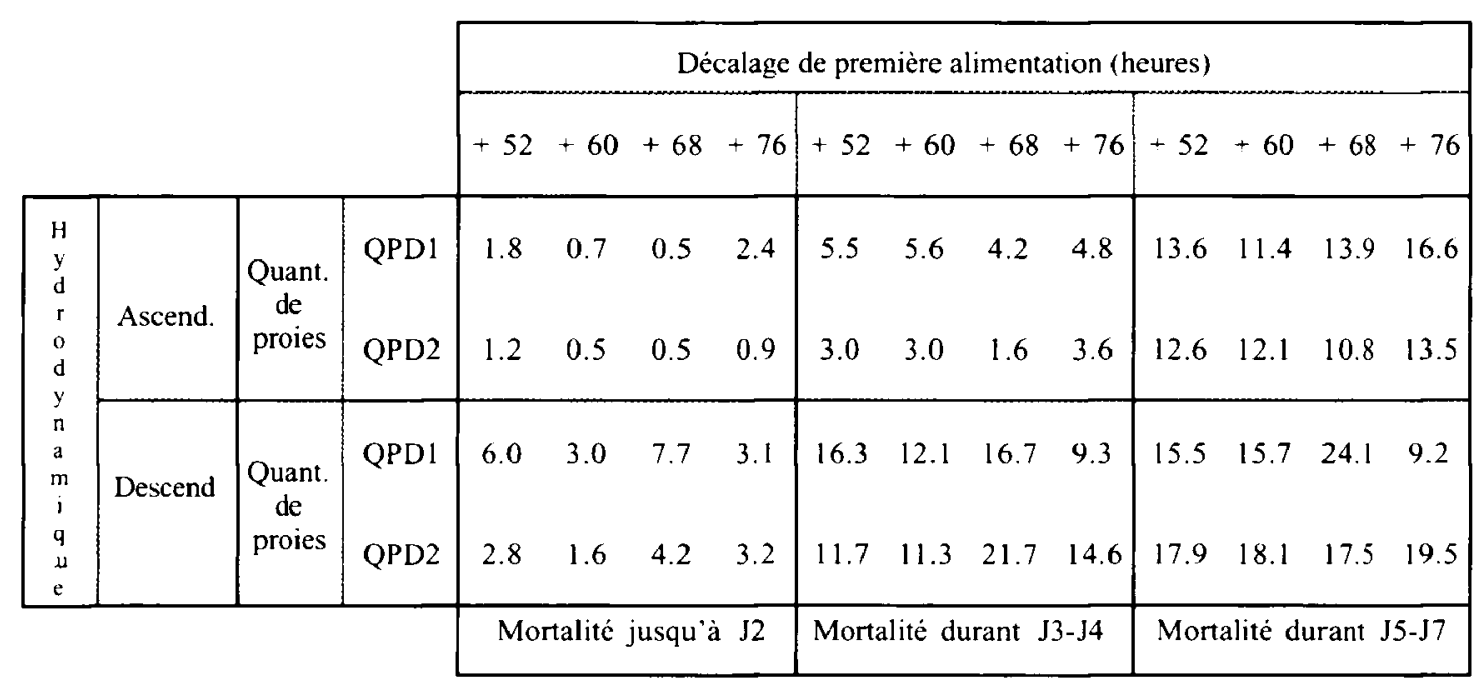

\begin{tabular}{|c|c|c|c|}
\hline Probabilité / sources de variation & Mortalité à J2 & Mortalité durant J3-J4 & Mortalité durant J5-J7 \\
\hline $\begin{array}{c}\text { Quantité de proies distribuées } \\
\text { (QPD) }\end{array}$ & 0.090 & 0.268 & 0.863 \\
$\begin{array}{c}\text { Décalage de la première distribution } \\
\text { alimentaire (DPA) }\end{array}$ & 0.277 & 0.524 & 0.839 \\
Hydrodynamique (H) & $\mathbf{0 . 0 0 8}$ & $\mathbf{0 . 0 0 2}$ & 0.104 \\
interactions entre facteurs & 0.404 & 0.255 & 0.489 \\
\hline
\end{tabular}




\section{Tableau IV}

Effets de l'Hydrodynamique dans les bassins (HA et HD), des Quantités de proies distribuées (QPD1 et QPD2) et du Décalage de la Première Distribution alimentaire (DPA) (de +52 à +76 heures après que $95 \%$ des larves soient écloses) sur la survie des larves de turbot (\%) en fin d'expérimentation (J7) (tableau du haut) avec résultats de l'analyse de variance (tableau du bas). Les chiffres en caractères gras indiquent les effets significatifs.

\section{Table IV}

Effects of tank hydrodynamics (HA, HD), feeding scheme (QPD1 or QPD2) and first feeding shift (DPA) (from +52 hours to +76 hours after $95 \%$ of the larvae hatched) on survival rate $(\%)$ of turbot larvae at the end of the experiment (J7) with 3way ANOVA results. Heavy types pointed out factors which have significant effects.

\begin{tabular}{|c|c|c|c|c|}
\hline & & & & $\begin{array}{l}\text { Décalage de première } \\
\text { alimentation (heures) }\end{array}$ \\
\hline & & & & $+52+60+68+76$ \\
\hline $\begin{array}{l}\text { H } \\
y \\
d \\
r \\
o \\
d\end{array}$ & Ascend. & $\begin{array}{l}\text { Quant. } \\
\text { de } \\
\text { proies }\end{array}$ & $\begin{array}{l}\text { QPD1 } \\
\text { QPD2 }\end{array}$ & $\begin{array}{llll}79.0 & 82.1 & 80.3 & 76.1 \\
83.1 & 84.4 & 87.1 & 82.0\end{array}$ \\
\hline $\begin{array}{l}\mathrm{a} \\
\mathrm{m} \\
\mathrm{i} \\
\mathrm{q} \\
\mathrm{u} \\
\mathrm{e}\end{array}$ & Descend & $\begin{array}{l}\text { Quant. } \\
\text { de } \\
\text { proies }\end{array}$ & $\begin{array}{l}\text { QPD1 } \\
\text { QPD2 }\end{array}$ & $\begin{array}{llll}62.1 & 69.0 & 51.4 & 78.3 \\
67.4 & 68.9 & 56.5 & 62.6\end{array}$ \\
\hline & & & & Survie à $\mathrm{J} 7$ \\
\hline
\end{tabular}

\begin{tabular}{|c|c|}
\hline Probabilité / sources de variation & Survie à J7 \\
\hline $\begin{array}{c}\text { Quantité de proies distribuées } \\
\text { (QPD) }\end{array}$ & 0.491 \\
$\begin{array}{c}\text { Décalage de la première distribution } \\
\text { alimentaire (DPA) }\end{array}$ & 0.428 \\
Hydrodynamique (H) & $\mathbf{0 . 0 0 6}$ \\
interactions entre facteurs & 0.310 \\
\hline
\end{tabular}




\section{Tableau V}

Effets de l'Hydrodynamique dans les bassins (HA et HD), des Quantités de proies distribuées (QPD1 et QPD2) et du Décalage de la Première Distribution alimentaire (DPA) (de +52 à +76 heures après que $95 \%$ des larves soient écloses) sur le poids $(\mu \mathrm{g})$ de larves de turbot à $\mathrm{J} 3, \mathrm{~J} 5$ et $\mathrm{J} 7$ (tableau du haut) avec résultats de l'analyse de variance (tableau du bas). Les chiffres en caractères gras indiquent les effets significatifs.

\section{Table V}

Effects of tank hydrodynamics (HA, HD), feeding scheme (QPD1 or QPD2) and first feeding shift (DPA) (from +52 hours to +76 hours after $95 \%$ of the larvae hatched) on larval weight $(\mu \mathrm{gg})$ of turbot larvae at $\mathrm{J3}, \mathrm{J} 5$ and $\mathrm{J7}$ with 3way ANOVA results. Heavy types pointed out factors which have significant effects.

\begin{tabular}{|c|c|c|c|c|c|c|c|c|c|c|c|c|c|c|c|}
\hline & & & & \multicolumn{12}{|c|}{ Décalage de première alimentation (heures) } \\
\hline & & & & +52 & +60 & +68 & +76 & +52 & +60 & +68 & +76 & +52 & +60 & +68 & +76 \\
\hline \multirow{2}{*}{$\begin{array}{c}\mathrm{H} \\
\mathrm{y} \\
\mathrm{d} \\
\mathrm{r} \\
\mathrm{o} \\
\mathrm{d} \\
\mathrm{y} \\
\mathrm{n} \\
\mathrm{a} \\
\mathrm{m} \\
\mathrm{i} \\
\mathrm{q} \\
\mathrm{d} \\
\mathrm{e}\end{array}$} & Ascend. & $\begin{array}{c}\text { Quant. } \\
\text { de } \\
\text { proies }\end{array}$ & QPD1 & $\begin{array}{l}220 \\
\pm 5 \\
222 \\
\pm 5\end{array}$ & $\begin{array}{l}225 \\
\pm 5 \\
226 \\
\pm 0\end{array}$ & $\begin{array}{l}225 \\
\pm 5 \\
228 \\
\pm 3\end{array}$ & $\begin{array}{l}212 \\
\pm 5 \\
221 \\
\pm 5\end{array}$ & $\begin{array}{l}244 \\
\pm 8 \\
268 \\
\pm 12\end{array}$ & $\begin{array}{l}216 \\
\pm 6 \\
246 \\
\pm 6\end{array}$ & $\begin{array}{l}228 \\
\pm 5 \\
226 \\
\pm 6\end{array}$ & $\begin{array}{l}236 \\
\pm 5 \\
243 \\
\pm 6\end{array}$ & $\begin{array}{l}375 \\
\pm 19 \\
366 \\
\pm 12\end{array}$ & $\begin{array}{l}386 \\
\pm 19 \\
469 \\
\pm 29\end{array}$ & $\begin{array}{l}248 \\
\pm 11 \\
483 \\
\pm 17\end{array}$ & $\begin{array}{l}359 \\
\pm 17 \\
302 \\
\pm 9\end{array}$ \\
\hline & Descend & $\begin{array}{c}\text { Quant. } \\
\text { de } \\
\text { proies }\end{array}$ & QPD 1 & $\begin{array}{l}214 \\
\pm 4 \\
216 \\
\pm 5\end{array}$ & $\begin{array}{l}215 \\
\pm 5 \\
208 \\
\pm 4\end{array}$ & $\begin{array}{l}200 \\
\pm 4 \\
205 \\
\pm 4\end{array}$ & $\begin{array}{l}210 \\
\pm 4 \\
209 \\
\pm 4\end{array}$ & $\begin{array}{l}246 \\
\pm 6 \\
254 \\
\pm 7\end{array}$ & $\begin{array}{l}203 \\
\pm 6 \\
275 \\
\pm 10\end{array}$ & $\begin{array}{l}224 \\
\pm 6 \\
\\
256 \\
\pm 7\end{array}$ & $\begin{array}{l}228 \\
\pm 4 \\
\\
284 \\
\pm 7\end{array}$ & $\begin{array}{l}372 \\
\pm 17 \\
416 \\
\pm 24\end{array}$ & $\begin{array}{l}279 \\
\pm 14 \\
392 \\
\pm 22\end{array}$ & $\begin{array}{l}310 \\
\pm 13 \\
428 \\
\pm 19\end{array}$ & $\begin{array}{l}254 \\
\pm 12 \\
408 \\
\pm 20\end{array}$ \\
\hline & & & & & oids & $\mathrm{J} 3(\mathrm{~m}$ & & & Poids & $\mathrm{J} 5$ & & & Poids à & J7 (mg & \\
\hline
\end{tabular}

\begin{tabular}{|c|c|c|c|}
\hline Probabilité / sources de variation & Poids à J3 & Poids à J5 & Poids à J7 \\
\hline $\begin{array}{c}\text { Quantité de proies distribuées } \\
\text { (QPD) }\end{array}$ & 0.357 & 0.031 & 0.085 \\
$\begin{array}{c}\text { Décalage de la première distribution } \\
\text { alimentaire (DPA) }\end{array}$ & 0.246 & 0.329 & 0.699 \\
Hydrodynamique (H) & 0.008 & 0.359 & 0.668 \\
interactions entre facteurs & 0.311 & 0.478 & 0.651 \\
\hline
\end{tabular}




\section{DISCUSSION}

Nos résultats montrent que tous les facteurs qui influencent l'hydrodynamique dans les bassins d'élevage, c'est-à-dire toutes les modifications qui peuvent être associées aux débit d'eau, débit d'air et de sens de circulation de l'eau ont une influence sur les survies larvaires chez le turbot durant les dix premiers jours d'élevage post-éclosion. Les effets observés dépendent cependant du stade de développement.

Le sens de circulation de l'eau apparaît comme ayant un effet prépondérant sur la survie durant toute la phase d'élevage étudiée et une inversion du sens de courant dans les bassins (quel que soit son sens et le moment où elle est pratiquée) entraîne une modification importante de l'évolution de la mortalité. Les meilleures performances sont obtenues avec un courant ascendant. Des résultats du même type ont déjà été observés par OLESEN et MINCK en 1983. Les effets observés apparaissent dès l'éclosion et avant même la première alimentation exogène, ils se poursuivent après la période d'alimentation mixte, et sont rapides, se traduisant lors d'une inversion du sens durant la période d'élevage par des conséquences quasi instantanées en termes de mortalité, y compris des arrêts de la mortalité. Les deux traitements mis en oeuvre ("HD» et "HA») sont cependant des systèmes complexes dans la mesure où les caractéristiques les différenciant sont nombreuses, d'autant plus que l'hydrodynamique dépend de très nombreux paramètres pouvant interagir. Ainsi, le renouvellement d'eau est supérieur dans le système d'eau ascendant comparé à celui utilisé dans le système descendant. Il est donc possible que la qualité de l'eau soit améliorée. Ceci en particulier car, dans le système ascendant, il n'existe pas de volumes d'eau morts. Des résultats non publiés montrent cependant qu'une réduction du débit dans ce système ne provoque pas d'accroissement de la mortalité jusqu'à J5. Par ailleurs, des travaux ont montré une interaction entre l'hydrodynamique et la disponibilité en proies (PERSON-LE RUYET, 1989 ; GULBRANDSEN, 1991, 1993 ; VAN DER MEEREN, 1991 ; SKRESLET, 1989). Les résultats de l'expérience 6 montrent cependant que la survie jusqu'à la fin de la période d'alimentation mixte dépend davantage du sens de circulation de l'eau que des méthodes d'alimentation. Comme l'ont déjà démontré IGLESIAS et al. (1994) chez le turbot ainsi que YUFERA et al. (1993) chez Sparus aurata, le décalage de la première alimentation n'influence ni la survie ni la croissance des larves. De plus, ni pour la survie, ni pour la croissance, n'ont été observées d'interactions entre les conditions hydrodynamiques et les différentes techniques d'alimentation essayées.

La survie des larves nourries dépend également du débit d'air. Mais les conditions les moins turbulentes, correspondant à la plus faible aération, apparaissent comme étant les plus défavorables et entraînent les plus fortes mortalités. Lorsque le milieu est fortement brassé par une forte aération, une meilleure disponibilité en proies liée à une absence de stratification des proies ne semble toutefois pas apparaître.

Durant les dix premiers jours de l'élevage des larves, après l'éclosion, l'effet hydrodynamique majeur observé semble donc être lié au sens ascendant de circulation de l'eau, qu'il soit dû à une aération ou au courant d'eau lui-même.

Chez les larves de turbot, la vision est considérée essentielle dans l'alimentation des larves (NEAVE, 1984 ; HOLMES et GIBSON, 1986 ; HUSE, 1993, 1994). Les distributions spatiales respectives des larves et des proies dépendent de l'hydrodynamique. En fonction des conditions d'élevage, les contrastes de lumière peuvent être modifiés ainsi que la perception qu'ont les larves des proies (GULBRANDSEN, 1993) et la possibilité de rencontre entre proies et larves (VAN DER MEEREN, 1991). Durant la phase d'intense activité de la larve (J3-J4) (SKIFTESVIK, 1992), le système de circulation d'eau ascendant pourrait faciliter la colonisation de tout le volume d'élevage, ce qui favoriserait l'accessibilité aux proies et ainsi offrirait de meilleures conditions aux larves pour survivre plus longtemps. 
Cependant, dans la mesure où nous n'observons pas d'interactions entre les facteurs hydrodynamiques et les aspects alimentaires étudiés, en particulier la densité en proies, cette hypothèse apparaît peu probable.

La flottabilité des larves dépend de la gouttelette lipidique jusqu'à la fin de la période mixte d'alimentation, puis ensuite de l'inflation de la vessie natatoire qui est fonctionnelle à partir de J3 ou J4 chez le turbot (COUSIN et BAUDIN-LAURENCIN, 1985). En facilitant le maintien de la position de la larve (BLAXTER, 1986), un courant ascendant pourrait influencer les coûts énergétiques liés à la nage et aux déplacements. Les survies prolongées chez les larves à jeun dans le cas de la plus forte aération semblent conforter cette hypothèse. Des résultats non publiés montrent également que la présence ou l'absence d'aération dans un système à circulation ascendante n'a pas d'effet sur la mortalité et ne permet pas d'expliquer les effets observés par des turbulences ou des chocs mécaniques. Un courant descendant pourrait donc augmenter la demande énergétique pour que les larves puissent se maintenir dans le volume d'eau alors que les besoins nutritionnels de croissance (fig. 4C) ne semblent pas être satisfaits dans nos expérimentations, et ainsi conduire à une augmentation de la mortalité. Le doublement du rationnement, s'il améliore la croissance dans l'expérience 6 et confirme que la densité de proies dans nos expériences est très faible comparée à celle couramment utilisée (6 à 60 rotifères/ml ; PERSON-LE RUYET, 1989), n'a cependant pas d'influence sur la survie larvaire.

Dans ce contexte, l'étude du débit d'eau a été réalisée avec un sens de circulation de l'eau descendant, donc dans un environnement défavorable. Même si l'on n'observe pas de différence de survie entre des débits constants lorsque les larves sont nourries, et ce malgré la différence de densité de proies disponibles liée au renouvellement d'eau, les survies initiales de larves à jeun sont prolongées pour le plus faible débit d'eau. De moindres turbulences liées à de plus faibles débits d'eau semblent donc correspondre à une situation plus confortable pour l'animal. Dans la même gamme de débits d'eau, une mortalité significative apparaît au jour 3 lorsqu'une variation de débit de $25 \%$ est provoquée. Cet effet ne se reproduit pas les jours suivants bien qu'un accroissement du débit soit également quotidiennement pratiqué. Dans les conditions d'environnement dans lesquelles se trouvent les larves, l'énergie supplémentaire requise pour faire face à cette variation est probablement trop importante et trop aiguë à ce moment critique de la période mixte d'alimentation lorsque la consommation d'oxygène de la larve augmente, l'absorption du globule lipidique est maximale, l'activité de la larve augmente rapidement (KAMLER, 1992 ; RONNESTAD et al., 1992 ; SKIFTESVIK, 1992), et a pu entraîner leur mort.

\section{CONCLUSION}

Nous pouvons conclure que les mortalités précoces observées chez le turbot ne sont probablement pas dues uniquement à la qualité des oeufs ou au stress de manipulation comme suggéré par MINKOFF et BROADHURST (1994). L'hydrodynamique dans les bassins, et en particulier le sens de circulation de l'eau (ascendant/descendant), est un facteur important influençant la survie larvaire chez le turbot.

De petites différences d'activité liées à l'hydrodynamique ou à l'activité natatoire peuvent probablement entraîner des consommations énergétiques considérables et conduire à des mortalités rapides, particulièrement sur les larves à jeun. Une telle dépendance des survies larvaires à de petites variations des débits d'alimentation en eau et en air pourrait expliquer une partie de la variabilité enregistrée couramment sur la survie des larves de turbot (STOTTRUP, 1993). Ceci justifie qu'une attention particulière soit portée à ces facteurs dès lors que l'on souhaite avoir un meilleur contrôle de la variabilité résiduelle en expérimentation. 
Toutefois, si l'on peut considérer que le rôle de l'alimentation semble peu important dans les effets de l'hydrodynamique, l'approche zootechnique mise en oeuvre ne permet pas d'appréhender précisément les mécanismes qui peuvent expliquer les résultats obtenus et en particulier la rapidité des réponses enregistrées lors des inversions de sens du courant qui n'ont été appréhendées ici ni au niveau physiologique, ni du comportement.

\section{RECOMMANDATIONS PRATIQUES}

Les expériences réalisées permettent de recommander pour la mise en oeuvre des dix premiers jours d'élevage larvaire du turbot:

- un contrôle le plus fréquent possible des débits d'eau et d'air permettant une stabilité dans le temps des paramètres d'élevage, et des conditions hydrodynamiques strictement identiques entre les bassins afin de limiter la variabilité des performances entre les lots :

- l'utilisation de courant d'eau ascendant permettant de meilleures survies (débit d'eau $1 \mathrm{l} / \mathrm{mn}$ pour des bassins cylindro-coniques de $150 \mathrm{l}$ );

- en cas d'utilisation de courant d'eau descendant, une aération de $30 \mathrm{ml} / \mathrm{mn}$ (pour les mêmes bassins).

\section{ANNEXE}

\section{Méthode de calcul de la mortalité}

Le principe de base de cette méthode est de déterminer un taux de mortalité pour une période donnée $t$ que l'on applique à la survie restante à $t-1$, ceci de $t=1$ à $t=f=f i n$ de l'expérience.

L'originalité de la méthode est de permettre d'intégrer:

1. les prélèvements réalisés sans remises (pour pesées, analyses, ...) :

Le calcul du taux de mortalité pour une période donnée $t$ est le rapport entre le nombre d'individus morts durant la période et l'effectif présent en début de période, soit à la fin de $\mathrm{t}-1$.

2. les individus manquants en fin d'expérience :

Après plusieurs expérimentations méthodologiques, nous avons été amenés à considérer que le nombre de larves manquantes n'était pas la conséquence d'erreurs liées à la distribution des larves en début d'expérience mais la conséquence des effets de techniques d'élevage particulières. En effet, certaines conditions hydrauliques ne permettent pas une décantation des larves mortes, nécessaire à leur récolte. Il s'en suit une lise post-mortem.

Le nombre d'individus manquants en fin d'expérience est la différence entre le nombre de larves au départ et la somme des larves mortes, des larves prélevées, des larves restantes en fin d'expérience. On en déduit un pourcentage de larves disparues que l'on intégrera à la mortalité enregistrée par l'intermédiaire d'un coefficient de proportionalité. 


\section{Symbole et abréviation}

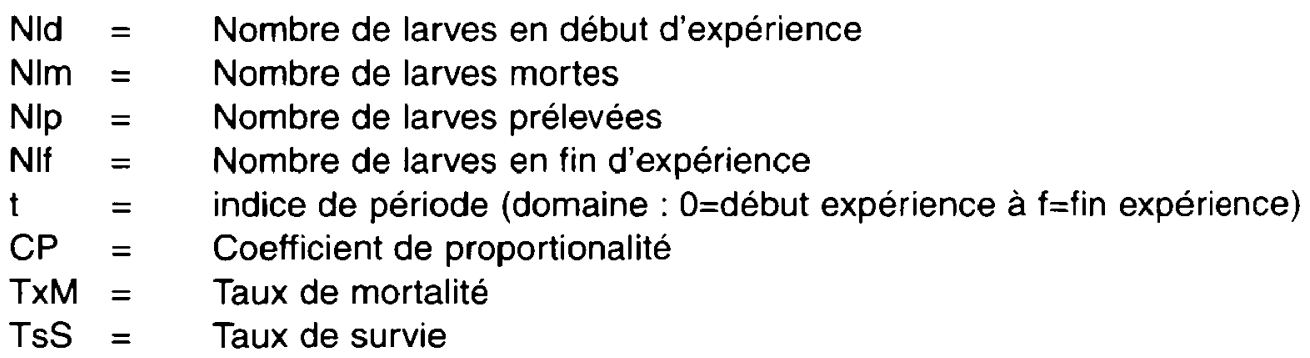

\section{Formulaire}

1. Détermination du Coefficient de proportionalité CP

$$
C P=\frac{N l d-\sum_{i=0}^{i=f} N l m-\sum_{t=0}^{t=f} N l p-N l f}{\sum_{i=0}^{t=f} N l m}
$$

2. Détermination du taux de mortalité à un instant $t$

$$
\begin{aligned}
& T x M_{t}=\frac{N l m_{t} \times(1+C P)}{N l d-\sum_{0}^{t-1}[(N l m \times(1+C P))+N l p]} \\
& \text { à } \mathrm{t}=1 \rightarrow \quad T x M_{1}=\frac{N l m_{1} \times(1+C P)}{N l d}
\end{aligned}
$$

3. Détermination du taux de survie

$$
\begin{aligned}
& T x S_{t}=T x S_{t-1} \times\left(1-T x M_{t}\right) \\
& \text { à } \mathrm{t}=1 \rightarrow T x S_{1}=1-T x M_{1}
\end{aligned}
$$

\section{BIBLIOGRAPHIE}

BERGH O., HANSEN G.H., JELMERT A., SKIFTESVIK A.B. \& TAXT R.E., 1991. Bacterial diseases of eggs and yolk sac larvae of halibut (Hippoglossus hippoglossus L.). LARVI'91. Fish and crustacean larviculture symposium. Lavens P., Sorgeloos P., Jaspers E. and Ollevier F. (eds.), European Aquaculture Society, Special Publication N¹5, Gent, Belgium.

BLAXTER J.H.S., 1986. Development of sense organs and behavior of teleost larvae with special reference to feeding and predator avoidance. Trans. am. fish. soc., 115, 98114.

CHESNEY E.J., Jr., 1989. Estimating the food requirements of striped bass larvae Morone saxatilis : effects of light, turbidity and turbulence. Mar. Ecol. Prog. Ser., 53, 191200. 
COUSIN J.C.B. \& BAUDIN-LAURENCIN L., 1985. Morphogenèse de l'appareil digestif et de la vessie gazeuse du turbot, Scophthalmus maximus L.. Aquaculture, 47, 305319.

CRIPPS S.J. \& POXTON M.G., 1992. A review of the design and performance of tanks relevant to flatfish culture. Aqu. Eng., 11, 71-91.

GATESOUPE F.J., 1990. The continuous feeding of turbot larvae, Scophthalmus maximus, and control of the bacterial environment of rotifers. Aquaculture, 89, 139148.

GULBRANDSEN J., 1991. Functional response of Atlantic halibut larvae related to prey density and distribution. Aquaculture, 94, 89-98.

GULBRANDSEN J., 1993. Prey consumption of Atlantic halibut (Hippoglossus hippoglossus L.) larvae : selectivity or availability ? Aquaculture, 116, 159-170.

HERNANDEZ-CRUZ C.M., SALHI M., FERNANDEZ-PALACIOS H. \& IZQUIERDO M.S., 1994. Improvements in the culture of Sparus aurata $L$. larvae in relation to the use of antibiotics, phytoplankton and rearing system. Aquaculture, 124, 269-274.

HOLMES R.A. \& GIBSON R.N., 1986. Visual cues determining prey selection by the turbot, Scophthalmus maximus L.. J. Fish Biol. (supplement A), 49-58.

HUSE I., 1993. First feeding larval sensory perception and behavioral programming implications for systems and procedures culture. Physiological and biochemical aspects of fish development. Walther and Fyhn Ed., 146-152.

HUSE I., 1994. Feeding at different illumination levels in larvae of three marine teleost species : cod, Gadus morhua L., plaice, Pleuronectes platessa L., and turbot, Scophthalmus maximus L.. Aqua. and fish. managt., 25, 687-695.

IGLESIAS J., RODRIGUEZ-OJEA G., ALVAREZ-BLAZQUEZ B. \& GOMEZ C., 1994. Definition of the point of no return in turbot (Scophthalmus maximus L.) larvae under starving conditions at three different temperatures. Lavens $P$. and Remmerswaal R.A.M. (eds.), European Aquaculture Society, Special Publication N²2, Gent, Belgium.

KAMLER E., 1992. Early life history of fish : an energetics approach. Chapman \& Hall, Fish and Fisheries Series 4, $267 \mathrm{p}$.

KILLGORE K.J., MILLER A.C. \& CONLEY K.C., 1987. Effects of turbulence on yolk-sac larvae of paddlefish. Trans. Amer. Fish Soc., 116(4), 670-673.

LECLERCQ D., 1994. Turbot broodstock management : a key point to mid-term progress of the turbot industry. Lavens P. and Remmerswaal R.A.M. (eds.), European Aquaculture Society, Special Publication No22, Gent, Belgium.

MINKOFF G. \& BROADHURST A.P., 1994. Intensive production of turbot, Scophthalmus maximus, fry. Lavens P. and Remmerswaal R.A.M. (eds.), European Aquaculture Society, Special Publication N²2, Gent, Belgium.

NEAVE D.A., 1984. The development of the retinomotor reactions in larval plaice (Pleuronectes platessa L.) and turbot (Scophthalmus maximus L.). J. Exp. Mar. Biol. Ecol., 76, 167-175.

OLESEN J.O. \& MINCK F., 1983. A technical solution to the mass-culturing of larval turbot. Aqua. Eng., 2, 1-12.

PERSON-LE RUYET J., 1989. The hatchery rearing of turbot larvae (Scophthalmus maximus). Cuadernos da Area de Ciencias Marinas, Seminario de Estudos Galegos, 3, 57-91.

RONNESTAD I., FYHAN H.J. \& GRAVNINGEN K., 1992. The importance of free amino acids to the energy metabolism of eggs and larvae of turbot (Scophthalmus maximus). Mar. Biol., 114, 517-525. 
SKIFTESVIK A.B., 1992. Changes in behaviour at onset of exogenous feeding in marine fish larvae. Can. J. Fish. Aquat. Sci., 49, 1570-1572.

SKRESLET S., 1989. Spatial match and mismatch between larvae of cod (Gadus morphua L.) and their principal prey, nauplii of Calanus finmarchicus (Gunnerus). Rapp. P.V. Réun. Cons. int. Explor. Mer., 191, 258-263.

STOTTRUP J.G., 1993. First feeding in marine fish larvae : nutritional and environmental aspects. Physiological and biochemical aspects of fish development. Walther and Fyhn Ed.

TANDLER A. \& HELPS S., 1985. The effects of photoperiod and water exchange rate on growth and survival of gilthead sea bream (Sparus aurata, Linnaeus ; Sparidae) from hatching to metamorphosis in mass rearing systems. Aquaculture, 48, 71-82.

VAN DER MEEREN T., 1991. Selective feeding and prediction of food consumption in turbot larvae (Scophthalmus maximus L.) reared on the rotifer Brachionus plicatilis and natural zooplankton. Aquaculture, 93, 35-55.

YUFERA M., PASCUAL E., POLO A. \& SARASQUETE M.C., 1993. Effect of starvation on the feeding ability of gilthead sea bream (Sparus aurata L.) larvae at first feeding. J. Exp. Mar. Biol. Ecol., 169, 259-272. 